\title{
Selenonium ylides: synthesis, characterization, and applications to photo-induced cyclopropanation reactions
}

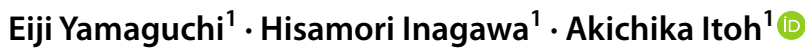

Received: 25 October 2021 / Accepted: 1 December 2021 / Published online: 20 January 2022

(c) The Author(s), under exclusive licence to European Photochemistry Association, European Society for Photobiology 2021

\begin{abstract}
Carbenes are important and highly reactive intermediates for the synthesis of various complex molecules. They are now an indispensable chemical species in organic chemistry and are used frequently to synthesize complex compounds in drug discovery chemistry. In general, carbenes are synthesized by a combination of transition metal catalysts and diazo compounds or by the decomposition reactions of diazo compounds. This paper reports the development of the visible light for the photochemical generation of carbenes from a novel $C, S e$-selenonium ylide. Overall, this photochemical carbene generation method using $C, S e$-selenonium ylide does not require a catalyst, is simple to perform, and enables highly efficient cyclopropanation reactions with alkenes.
\end{abstract}

\section{Graphical abstract}

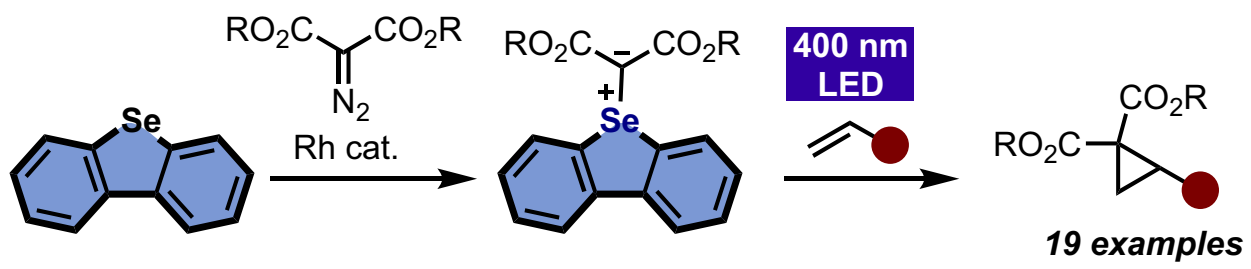

Keywords Carbene $\cdot$ Selenonium ylide $\cdot$ Cyclopropanation $\cdot$ Photoreaction

\section{Introduction}

Carbenes are a neutral, six-electron carbon species with unique reactivity [1]. These compounds have been actively studied in synthetic organic chemistry, and various reactions have been developed using them [2,2]. Although free carbenes are prepared by the thermal or photochemical decomposition of carbene precursors, control of their reactivity has significant drawbacks. Moreover, the reactivity and selectivity can be controlled by synthesizing metal carbenoids as free carbene precursors through a reaction of

Eiji Yamaguchi

Yamaguchi@gifu-pu.ac.jp

$\triangle$ Akichika Itoh

itoha@gifu-pu.ac.jp

1 Gifu Pharmaceutical University, 1-25-4 Daigaku-Nishi, Gifu, Japan diazo compounds with metal complexes [4]. Various metals, such as $\mathrm{Rh}, \mathrm{Cu}$, and $\mathrm{Pd}$, can be used to produce metal carbenoids. Considerable progress has been made in elucidating the reactivity depending on the type of diazo compound and metal [2-4]. Accordingly, various reactions can be carried out using carbenes. Carbenes are a reactive chemical species essential in the total synthesis and construction of complex molecules [7]. Although diazo compounds are often used to generate metal carbenoids because of their high efficiency, there are significant problems for their large-scale applications in terms of safety and handling. Therefore, the use of main group element ylides as alternative reagents has attracted attention $[5-8,10,13]$.

In 2007, Jenks et al. developed $C, S$-sulfonium ylides based on the thiophene moiety as a useful precursor for carbenes using a photochemical activation methodology (Scheme 1a) [9]. The synthesized sulfonium ylides were 
Scheme 1. a Jenks' sulfonium ylide strategy to generate carbenes; (b) molecular design for novel ylide; (c) methodology for visible-light-induced carbene generation a: Jenks' sulfonium ylide

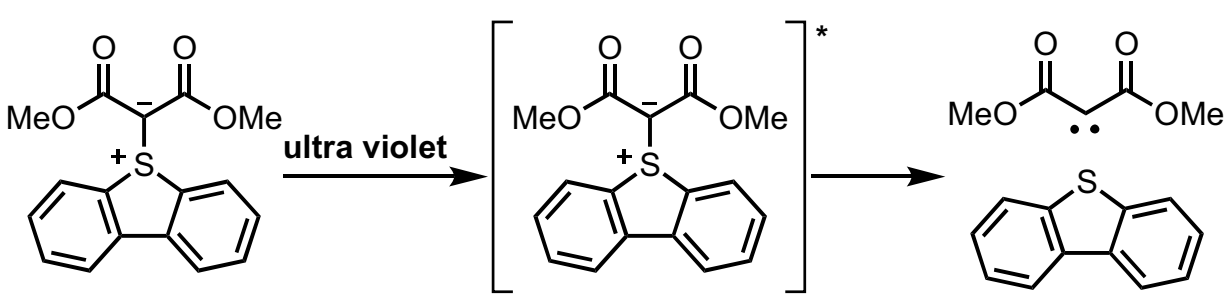

b: Our molecular design for photo-responsive onium ylide
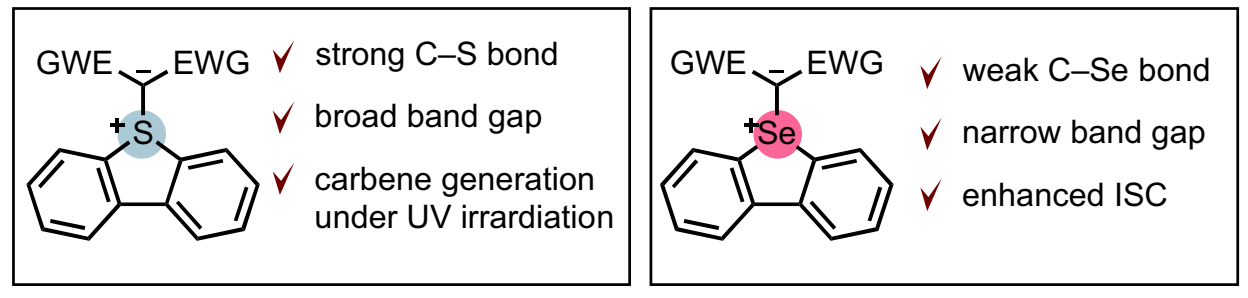

c: This work: Visible light induced carbene generation from selenonium ylide<smiles>[R]OC(=O)/C(=C/C(=O)O)[Se]1(C)c2ccccc2-c2ccccc21</smiles>
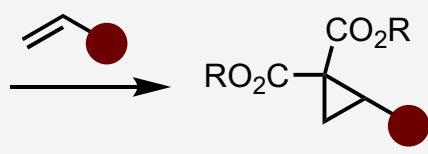

stable, easy to handle, and could generate free carbenes using ultraviolet (UV) irradiation without a catalyst [10, $15,17]$. These studies reported that onium ylides with relatively large conjugated systems could photochemically generate the corresponding carbene species. Furthermore, the carbenes released from these onium ylides require intense UV light. Hence, improvements are needed before they can be implemented in synthetic organic chemistry. Therefore, this study developed a highly versatile carbene precursor that can release carbenes under visible-light irradiation and milder conditions. The necessity of UV light for the excitation wavelength in $C, S$-sulfonium ylides was attributed to the $\mathrm{C}-\mathrm{S}$ bond strength and the absorption wavelength of dibenzothiophene [11, 19]. Accordingly, using this ylide motif, selenium, a member of the same group as sulfur, was chosen because it can form weaker bonds than sulfur with carbon. Selenium has a larger atomic radius than sulfur. Additionally, compounds containing selenium have a narrower HOMO-LUMO gap, and the heavy atom effect stabilizes the excited triplet state (Scheme 1b) [12, 13, 21]. Therefore, it was hypothesized that the corresponding selenonium ylides could be excited by visible light to produce the carbene species efficiently. Thus, $C$,Se-selenonium ylides were synthesized, and their physical properties and reactivities as carbene precursors were evaluated. This paper reports the synthesis and characterization of novel $C$, Se-selenonium ylides and their applications to the visible-light-responsive radical generation reaction (Scheme 1c).

\section{Results and discussion}

\subsection{Synthesis of dibenzoselenophene-based selenonium ylide}

The synthesis of the selenonium ylide was started with a reaction of diazomalonate 2 with dibenzoselenophene (Table 1) [23]. After the optimization study, the desired dibenzoselenophene-based selenonium ylide $3 \mathbf{a}$ was obtained in good yield through a rhodium acetate dimercatalyzed carbenoid addition reaction with selenophene [14]. The corresponding dibenzylmalonate derivative $\mathbf{3 b}$ was also prepared under the optimized reaction conditions. The desired ylide using dibenzoselenophene was synthesized with electron-withdrawing or electron-donating substituents to control the absorption wavelength of selenonium ylide. Both reactions gave the corresponding products (3b and $\mathbf{3 c}$ ) without any loss of yield. The ylidization reaction also proceeded under these conditions for compounds with extended conjugation systems, giving the corresponding products in moderate yield. 
Table 1 Synthesis of selenonium ylide $\mathbf{3}^{\mathrm{a}}$

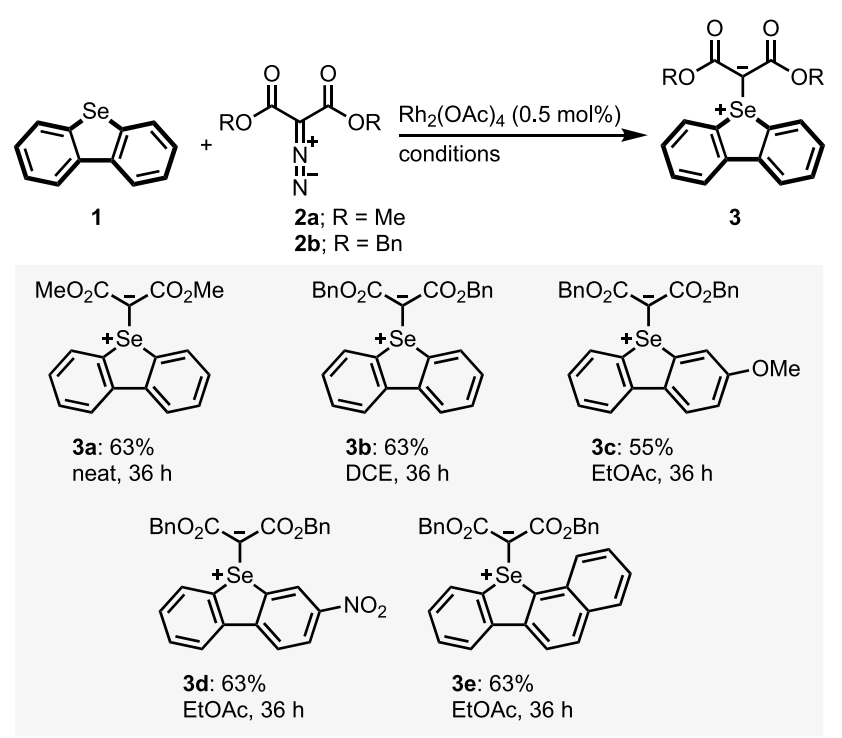

${ }^{a}$ Isolated yield.

\subsection{UV/Visible spectra of selenonium ylide 3}

A series of selenonium ylides $\mathbf{3}$ were synthesized and their photochemical properties were assessed to realize the photochemical carbine-formation reaction using $\mathbf{3}$. Figure 1 shows the spectra of the measured compounds.

Photophysical studies showed that the substituents have a significant influence on absorption (Table 2). The $\lambda_{\text {abs }}$ values of selenonium ylide-bearing electron-donating groups on the dibenzoselenophen ring, such as $\mathbf{3 c}$ (276, 298, and

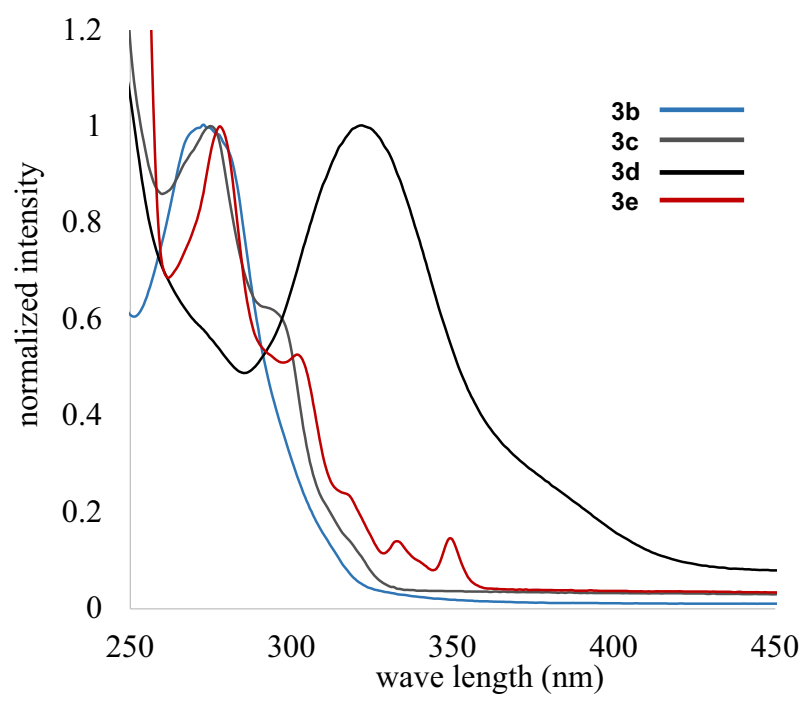

Fig. 1 Ultraviolet/Visible spectra of the selenonium ylide $3^{\text {a }} .{ }^{\text {a }}$ Measured in $\mathrm{MeCN}$
Table 2 Ultraviolet/Visible absorption of selenonium ylide $\mathbf{3}$

\begin{tabular}{llll}
\hline Entry & Ylides & UV/Vis & \\
\cline { 3 - 4 } & & $\lambda_{\max }(\mathrm{nm})$ & $\log \varepsilon$ \\
\hline 1 & 3b & 274 & 4.43 \\
& & $330(\mathrm{sh})$ & 3.00 \\
& 3c & 276 & 4.25 \\
& & 298 & 4.03 \\
& & $320(\mathrm{sh})$ & 3.32 \\
& 3d & 275 & 3.91 \\
& & 325 & 4.15 \\
4 & & $385(\mathrm{sh})$ & 3.53 \\
& & 280 & 4.21 \\
& & 304 & 3.92 \\
& & 320 & 3.54 \\
& & 334 & 3.34 \\
& & 350 & 3.37 \\
\hline
\end{tabular}

$320 \mathrm{~nm}$ ), revealed a similar absorption spectrum to those of the parent, unsubstituted ylide $\mathbf{3 b}(274,330 \mathrm{~nm})$. Those with electron-withdrawing substituents, such as $\mathbf{3 d}(275,325$, and $385 \mathrm{~nm}$ ), were red-shifted significantly (entries 1-3). Furthermore, the UV/visible absorption spectra of $\mathbf{3 e}$, a compound with a benzo[b]naphtho[2, $1-d]$ selenophene system, showed complex absorption spectra derived from the mother framework. On the other hand, the strong absorption peak at $280 \mathrm{~nm}$, which was attributed to the ylide, showed no shift. The shoulder peaks of the absorption spectra of selenonium ylide 3 were strong enough in the visible-light region to be considered for photochemical studies using $\mathbf{3}$.

\subsection{Optimization of the reaction condition for the cyclopropanation of styrene with selenonium ylide 3}

Based on the results described above, attention was then focused on carbene generation using selenonium ylide $\mathbf{3}$ under visible-light irradiation (Table 3 ). The initial optimization study showed that a reaction of $\mathbf{3}$ with $\mathbf{4 a}$ (10 equiv) in $\mathrm{MeCN}$ as a solvent was crucial for the photo-induced generation of the desired carbine [25]. The reaction of the dimethyl malonate-derived selenium ylide $\mathbf{3 a}$ with styrene 4b under $365 \mathrm{~nm}$ LED irradiation for $20 \mathrm{~h}$ furnished the corresponding cyclopropane 5aa in 32\% NMR yield (entry $1)$. The reaction proceeded depending on the wavelength irradiated with the LED. The yield was improved greatly to $57 \%$ when irradiated with a longer wavelength, e.g., $400 \mathrm{~nm}$, while it decreased when irradiated with $420 \mathrm{~nm}$ LED radiation (entries 2 and 3). This trend was also observed for the dibenzyl ester derivative 3b, where 365-420 nm light gave the product $5 \mathrm{ba}$ with the highest NMR yield of 66\% (56\% isolated yield) for the $400 \mathrm{~nm}$ light source (entries 4-6). 
Table 3 Optimization study for the cyclopropanation of styrene $\mathbf{4 a}$ with $\mathbf{3}^{\mathrm{a}}$

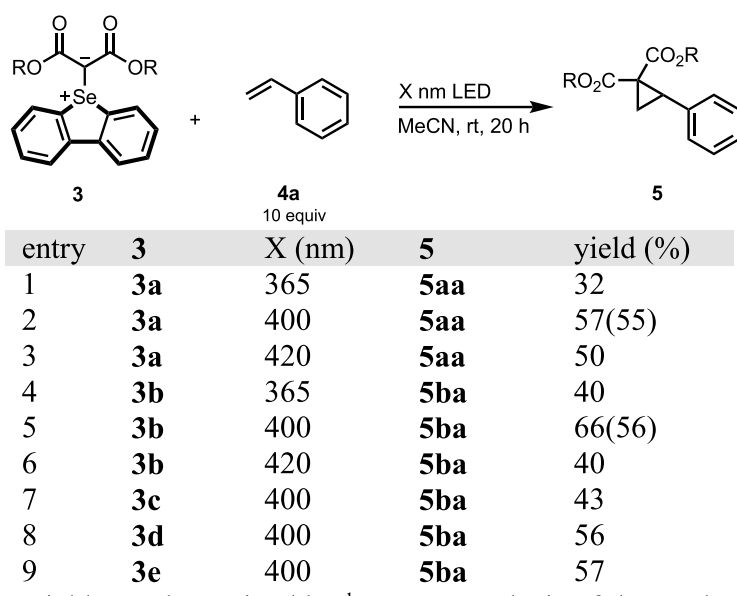

${ }^{\text {a }}$ Yield was determined by ${ }^{1} \mathrm{H}$ NMR analysis of the crude reaction mixture using 1,1,2,2-tetrachloroethane as an internal standard. The numbers in parentheses are the isolated yields.

However, the reaction proceeded in response to the shoulder peak, which is a weak absorption band because the maximum absorption wavelength was approximately $300 \mathrm{~nm}$. This reaction was also applied to various substituted compounds 3. No improvement in yield was observed using a dibenzoselenophene with an electron-donating functional group 3c (entry 7). On the other hand, the reaction using 3d, which has a more red-shifted absorption wavelength, gave the product in similar yield compared to the case of $\mathbf{3 b}$ (entry 8). Although the benzo[b]naphtho[2, $1-d]$ selenophene system-derived ylide was also applied to the carbene generation reaction, the desired cyclopropane 5 ba was obtained in $57 \%$ yield (entry 9). Overall, using $\mathbf{3 b}$ as a carbene precursor under $400 \mathrm{~nm}$ irradiation conditions is optimal for the present reaction.

\subsection{Substrate scope for the cyclopropanation of olefins with selenonium ylide 3}

Encouraged by the optimization study, the general applicability of this cyclopropanation reaction was evaluated, and the influence of a reaction partner was studied. Therefore, a set of differently substituted styrenes were first studied under optimized reaction conditions (Table 4, also see Tables S3-7 in ESI for full detailed substate scope.). When para-methoxy styrene $\mathbf{4 b}$ was used as a donor component, the desired product $\mathbf{5 b b}$ was isolated in a lower yield (29\%). The introduction of a para-bromo substituent (4c) led to a slight decrease in reactivity. In contrast, the developed cyclopropanation reaction using $\mathbf{3 b}$ proceeded in a good yield for the ortho $(\mathbf{4 d})$, meta (4e), and para (4f)-methyl substituent on styrene. In the case of the $t$-butyl group ( $\mathbf{4} \mathbf{g}$ ) and methyl group, the reaction proceeded smoothly, and the corresponding carbene adducts were obtained in moderate yield. This reaction was also applicable to vinyl naphthalenes, which are $\pi$-expanded aromatic carbons, showed a red shift in the absorption band, and furnished the corresponding products in moderate yield. The reactions between $\mathbf{3 b}$ and $\alpha$-olefins $\mathbf{4}$ were then examined. In this case, treatment of a mixture of $\mathbf{3 b}$ and 1 -decene $\mathbf{4 j}$ in $\mathrm{MeCN}$ under $400 \mathbf{n m}$ LED irradiation gave $\mathbf{5 b j}$ in $50 \%$ yield. The developed reaction condition using $\mathbf{3 b}$ was also applied to various olefins, such as those bearing a bromo group $\mathbf{4} \mathbf{k}$, sterically hindered substrate at the allyl position $\mathbf{5} \mathbf{~ l}$, methyl ester $\mathbf{5} \mathbf{~ m}$, and acetyl group 5n, and furnished the desired cyclopropanation product in moderate yield. Interestingly, this cyclopropanation reaction proceeded even in the presence of free hydroxyl groups, giving the corresponding products $\mathbf{3 b o}$ in low yields.

This process was extended to other unsaturated molecules, such as internal alkenes (Table 5). Interestingly, ethyl cinnamate (4p) gave the corresponding cyclopropane 5 bp in $37 \%$ yield with complete anti-diastereoselectivity. With other internal olefins, such as cyclohexene (3q) and cyclohexadiene (3r), the developed photo-induced cyclopropanation reaction proceeded smoothly to give the product in $43 \%$ and $30 \%$ yield, respectively. Interestingly, previous experiments on the generation of carbenes from thiophene-based ylides reported the carbene insertion into the allylic $\mathrm{C}-\mathrm{H}$ bond [15]. In the present reaction, however, the cyclopropanation reaction proceeded predominantly. This suggests that the singlet carbene is generated preferentially in the present system and that singlet to triplet intersystem crossing (ISC) does not occur [15]. Furthermore, norbornene (3 s) underwent the required transformation to furnish exo-cyclopropanation products in good yield.

\section{Conclusion}

An efficient synthetic method was developed to prepare selenonium ylides from readily available dibenzoselenophenes. The selenonium ylides prepared were excited 
Table 4 Reaction of various olefines 4 with the selenonium ylide $\mathbf{3} \mathbf{b}^{\mathrm{a}}$
Table 5 Reaction of various internal alkenes 4 with selenonium ylide $3 \mathbf{b}^{\mathrm{a}}$

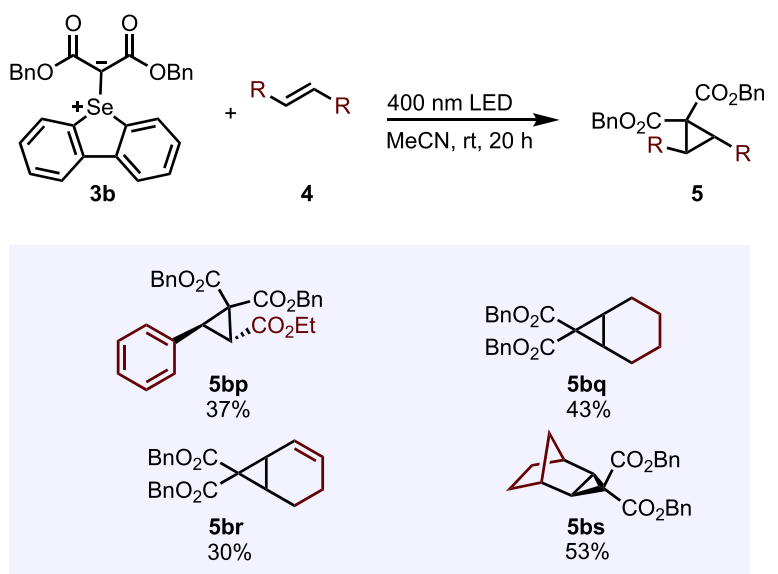

${ }^{a}$ Isolated yield.

by visible light without the need for sensitizers or photocatalysts to give the corresponding carbenes. In addition, this photo-induced carbene generation method from selenononium ylides revealed a reaction with various olefins to furnish the corresponding cyclopropane products.

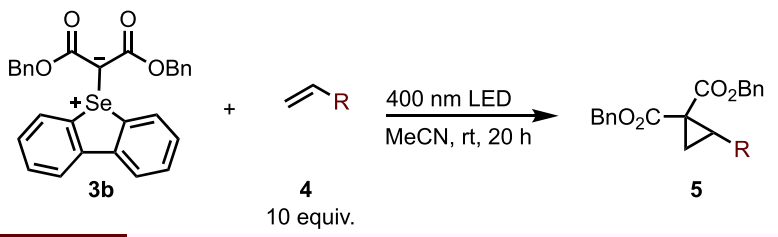

\section{Stryrenes}
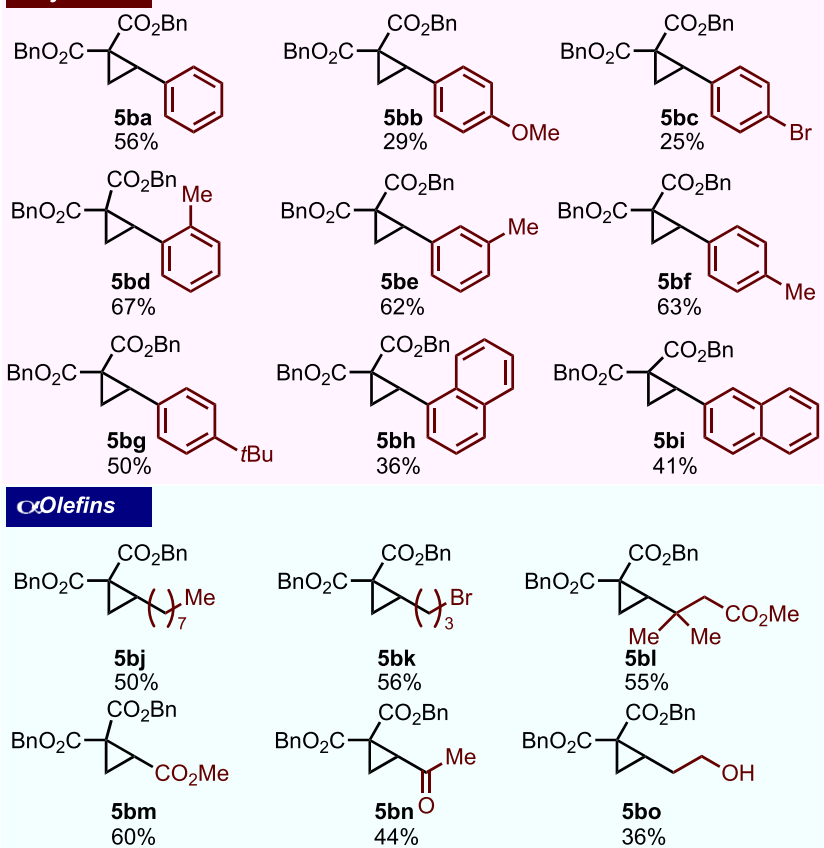<smiles>CC(Br)(Br)C1(C(=O)OBr)CC1(C(=O)OBr)C(=O)Oc1ccccc1</smiles>
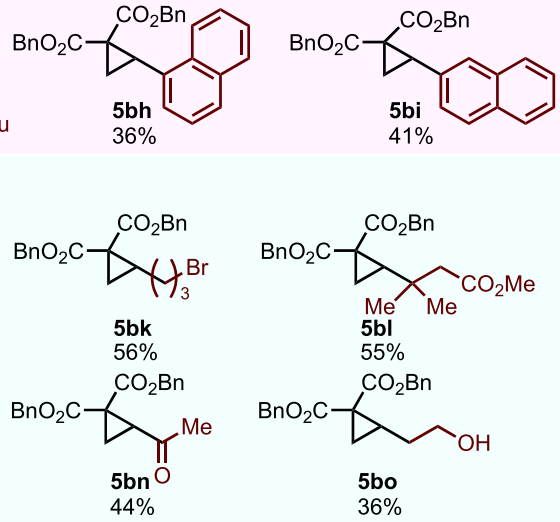

${ }^{a}$ Isolated yield.

The carbene species produced in this reaction were preferentially singlet carbenes, and the ISC did not proceed because of the reaction with visible light. The method of photochemically generating active species from such heavy main group element-based ylides is interesting. The synthesis of ylides for the generation of nitrenes is currently under investigation.

Supplementary Information The online version contains supplementary material available at https://doi.org/10.1007/s43630-021-00155-2.

Author contributions Conceptualization, EY; investigation, HI and $\mathrm{EY}$; supervision, AI and EY; writing-original draft preparation, AI, EY; funding acquisition: EY. All authors have read and agreed to the submitted version of the manuscript.

Funding This work was supported in part by JSPS KAKENHI Grant Number 20K06968.

\section{Declarations}

Conflict of interest The authors declare that they have no known competing financial interests or personal relationships that could have appeared to influence the work reported in this paper. 


\section{References}

1. Hahn, F. H. (2018). Introduction: carbene chemistry. Chemical Reviews, 118(19), 9455-9456.

2. Zhu, D., Chen, L., Fan, H., Yao, Q., \& Zhu, S. (2019). Recent progress on donor and donor-donor carbenes. Chemical Society Reviews, 49, 908-950. https://doi.org/10.1039/C9CS00542K

3. Frémonta, P., Marion, N., \& Nolan, S. P. (2009). Carbenes: synthesis, properties, and organometallic chemistry. Coordination Chemistry Review, 253(7-8), 862-892.

4. Davies, H. M. L., \& Beckwith, R. E. J. (2003). Catalytic enantioselective $\mathrm{C}-\mathrm{H}$ activation by means of metal-carbenoid-induced C-H insertion. Chemical Reviews., 103(8), 2861-2904. https:// doi.org/10.1021/cr0200217

5. Davies, H. M. L., \& Liao, K. (2019). Dirhodium tetracarboxylates as catalysts for selective intermolecular $\mathrm{C}-\mathrm{H}$ functionalization. Nature Reviews Chemistry., 3, 347-360.

6. Gillingham, D., \& Fei, N. (2013). Catalytic X-H insertion reactions based on carbenoids. Chemical Society Reviews, 42, 49184931. https://doi.org/10.1039/C3CS35496B

7. Davies, H. M. L., \& Hedley, S. J. (2007). Intermolecular reactions of electron-rich heterocycles with copper and rhodium carbenoids. Chemical Society Reviews, 36, 1109-1119.

8. Davies, H. M. L., \& Denton, J. R. (2009). Application of donor/ acceptor-carbenoids to the synthesis of natural products. Chemical Society Reviews, 38, 3061-3071. https://doi.org/10.1039/B9011 $70 \mathrm{~F}$

9. Mayakrishnan, S., Tamizmani, M., \& Maheswari, N. U. (2020). Harnessing hypervalent iodonium ylides as carbene precursors: $\mathrm{C}-\mathrm{H}$ activation of $\mathrm{N}$-methoxybenzamides with a $\mathrm{Rh}$ (iii)-catalyst. Chemical Communications., 56, 15462-15465. https://doi.org/10. 1039/D0CC06038K

10. Jiang, Y., Li, P., Zhao, J., Liu, B., \& Li, X. (2020). Iodonium ylides as carbene precursors in $\mathrm{Rh}(\mathrm{III})$-catalyzed $\mathrm{C}-\mathrm{H}$ activation. Organic Letters, 22(19), 7475-7479. https://doi.org/10.1021/acs. orglett.0c02618

11. Huang, Y., Lyu, X., Song, H., \& Wang, Q. (2019). Sulfoxonium ylides as carbene precursors: Rhodium(III)-catalyzed sequential $\mathrm{C}-\mathrm{H}$ functionalization, selective enol oxygen-atom nucleophilic addition, and hydrolysis. Advanced Synthesis and Catalysis., 361(22), 5272-5276. https://doi.org/10.1002/adsc.201900861

12. Xu, Y., Zhou, X., Zheng, G., \& Li, X. (2017). Sulfoxonium ylides as a carbene precursor in $\mathrm{Rh}$ (III)-Catalyzed $\mathrm{C}-\mathrm{H}$ acylmethylation of arenes. Organic Letters, 19(19), 5256-5259. https://doi.org/10. 1021/acs.orglett.7b02531

13. Shevchenko, I. V., Rogalyov, A. E., Poliakov, D. V., \& Röschenthaler, G. V. (2008). Ylides and carbenes: The first carbene catalyzed reaction. Phosphorus, Sulfur, and Silicon and the Related Elements., 183, 576-577. https://doi.org/10.1080/10426 500701764973

14. Camacho, M. B., Clark, A. E., Liebrecht, T. A., \& DeLuca, J. P. (2000). A phenyliodonium ylide as a precursor for dicarboethoxycarbene: Demonstration of a strategy for carbene generation. Journal of American Chemical Society., 122(21), 5210-5211. https://doi.org/10.1021/ja000334o
15. Stoffregen, S. A., Heying, M., \& Jenks, W. S. (2007). C, S-Sulfonium ylides from thiophenes: Potential carbene precursors. Journal of American Chemical Society., 129(51), 15746-15747. https://doi.org/10.1021/ja076351w

16. Jenks, W. S., Heying, M. J., Stoffregen, S. A., \& Rockafellow, E. M. (2009). Reaction of dicarbomethoxycarbene with thiophene derivatives. The Journal of Organic Chemistry., 74, 2765-2770. https://doi.org/10.1021/jo802823s

17. Jenks, W. S., Heying, M. J., \& Rockafellow, E. M. (2009). Modulation of carbene spin state population through precursor photophysics. Organic Letters., 11(4), 955-958. https://doi.org/10.1021/ ol802934w

18. Kolattoor, J., Sherman, M., \& Jenks, W. S. (2018). Product sensitization in the photochemical generation of carbenes from sulfonium ylides. Journal of Photochemistry and Photobiology A: Chemistry., 365, 208-212. https://doi.org/10.1016/j.jphotochem. 2018.07.016

19. McCulla, R. D., \& Jenks, W. S. (2004). Deoxygenation and other photochemical reactions of aromatic selenoxides. Journal of American Chemical Society., 126(49), 16058-16065. https://doi. org/10.1021/ja045935k

20. Stoffregen, S. A., Lee, S. Y., Dickerson, P., \& Jenks, W. S. (2014). Computational investigation of the photochemical deoxygenation of thiophene-S-oxide and selenophene-Se-oxide. Photochemical and Photobiological Sciences., 13, 431-438. https://doi.org/10. 1039/C3PP50382H

21. Huang, W., Wang, M., Hu, L., Wang, C., Xie, Z., \& Zhang, H. (2020). Recent advances in semiconducting monoelemental selenium nanostructures for device applications. Advanced Functional Materials., 30(42), 2003301. https://doi.org/10.1002/adfm.20200 3301

22 Schwartz, P.-O., Fortsch, S., Vogt, A., Mena-Osteritzl, E., \& Bäuerle, P. (2019). Selenophene-containing heterotriacenes by a C-Se coupling/cyclization reaction. Beilstein Journal of Organic Chemistry, 15, 1379-1393. https://doi.org/10.3762/bjoc.15.138

23. Fringuelli, F., Marino, G., Taticchi, A., \& Grandolini, G. (1974). A comparative study of the aromatic character of furan, thiophen, selenophen, and tellurophene. Journal of the Chemical Society, Perkin Transactions, 2, 332-337. https://doi.org/10.1039/P2974 0000332

24. See the Supporting information for full detailed study toward selenonium ylide.

25. Drabowicz, J., Rzewnicka, A., \& Żurawiński, R. (2020). Selenonium ylide selenonium ylides: syntheses, structural aspects, and synthetic applications. Molecules, 25(10), 2420. https://doi.org/ $10.3390 /$ molecules 25102420

26. See the Supporting information for full detailed study for optimization of reaction condition.

27. Drummond, B. H., Hoover, G. C., Gillete, A. J., Aizawa, N., Myers, W. K., McAllister, B. T., Jones, S. T. E., Pu, Y.-J., Credgington, D., \& Seferos, D. S. (2020). Selenium substitution enhances reverse intersystem crossing in a delayed fluorescence emitter. Journal of Physical Chemistry C, 124(11), 6364-6370. https://doi.org/10.1021/acs.jpcc.0c01499 